\title{
SPECT in focal epilepsies
}

\author{
Roderick Duncan \\ Institute of Neurological Sciences, Southern General \\ Hospital, Glasgow G51 4TF, Scotland
}

Brain perfusion changes during seizures were first observed in the 1930s. Single Photon Emission Computed Tomography (SPECT) was developed in the 1970s, and tracers suitable for the imaging of regional cerebral perfusion ( $\mathrm{rCP}$ ) became available in the 1980s. The method was first used to study $\mathrm{rCP}$ in the interictal phase, and this showed areas of low perfusion in a proportion of cases, mainly in patients with temporal lobe epilepsies. However, the trapping paradigm of tracers such as hexamethyl propyleneamine oxime (HMPAO) provided a practicable method of studying changes in $\mathrm{rCP}$ during seizures, and a literature was established in the late 1980s and early 1990s showing a typical sequence of changes during and after seizures of mesial temporal lobe origin; the ictal phase was associated with large increases in perfusion throughout the temporal lobe, with first the lateral, then the mesial temporal lobe becoming hypoperfused in the postictal phase. Activation and inhibition of other structures, such as the basal ganglia and frontal cortex, were also seen. Studies of seizures originating elsewhere in the brain have shown a variety of patterns of change, according to the structures involved. These changes have been used practically to aid the process of localisation of the epileptogenic zone so that epilepsy surgery can be planned.

Some neuroreceptors (e.g. benzodiazepine receptors) can be studied using SPECT, and have shown localised abnormalities. SPECT has also been used to study brain function during the intracarotid amytal test. SPECT images of all kinds can be analysed using numerical techniques such as statistical parametric mapping, and such techniques promise to improve the yield of information from ictal studies.

\section{Introduction}

The fact that seizures are associated with changes in cerebral perfusion has been known since the thirties. Wilder Penfield, a pioneering epilepsy surgeon working in Montreal, made the first observations in a remarkable circumstance [1-3]. Lacking any other localising techniques, he would open a craniotomy over the appropriate motor cortex in patients with Jacksonian seizures, and stimulate the cortex electrically until he found that part of it which could replicate the patient's habitual seizure. He was thus in a position to observe alterations of cortical perfusion directly, seeing either reddening or pallor, and described patterns of change which included hyperperfusion of the area of cortex involved in the seizure, and hypoperfusion during and after seizures, usually surrounding the hyperperfused area.

EEG became available in the thirties, and soon became the main localising tool for epilepsy surgeons, so observations of ictal perfusion changes lapsed until the advent of functional imaging techniques. PET was able to capture seizures fortuitously in a small number of patients, but the it was the development of the tracer HMPAO, with its novel trapping paradigm, which made SPECT a practical tool for studying the local and regional ictal and postictal changes in regional cerebral perfusion ( $\mathrm{rCP}$ ) which had first been observed by Penfield 50 years earlier.

The main impetus to study ictal and postictal changes in cerebral perfusion was the potential for such changes to help localise the source of seizures non-invasively, and thus to allow surgical resection. This remains the clinical use of ictal and postictal SPECT.

\section{Focal epilepsy}

\subsection{The interictal phase}

This article is mainly concerned with ictal and postictal changes in perfusion, but it also is worth considering perfusion abnormalities in the interictal state. The usual finding is regional hypoperfusion, which occurs most commonly in medial temporal lobe epilepsies, in approximately $50 \%-70 \%$ of patients [4-9]. The hypoperfusion usually involves all of the temporal lobe (Fig. 1), though isolated medial hypoperfusion is seen. The ipsilateral frontal lobe, occasionally the ipsilateral hemisphere may also be hypoperfused. Bilateral temporal hypoperfusion may occur.

The pathophysiological basis of interictal temporal hypoperfusion is uncertain. It is associated with epilepsy of early onset [10] and ipsilateral medial tem- 
poral sclerosis [11], and there appears to be a topographic relationship with interictal spiking recorded intracranially [12].

Some lesions are associated with focal hypoperfusion, as a simple consequence of their physical presence. Others, such as some tumours and vascular malformations have high HMPAO uptake [13]. Focal hyperperfusion may also occur in non lesional cases [7] possibly representing prolonged postictal hyperperfusion, which can last for some days [14].

In focal epilepsies originating outside the medial temporal lobe, interictal abnormalities of perfusion appear to be much less common, occurring in $10 \%$ or less of patients $[15,16]$.

\subsection{Ictal \& postictal rCP SPECT - their use in localising seizures}

Currently, the most confident localisation of an epileptogenic zone results from demonstrating a localised physiological change which takes place in temporal association with the clinical event (i.e. the seizure) to be treated. It is patently difficult to image the brain during a clinical event which is brief, unpredictable and involves movement, but the trapping paradigm of tracers such as HMPAO (and more recently ECD) allows just that $[17,18]$. The tracer is a lipophilic material which can be injected intravenously. It can cross the blood-brain barrier, and is distributed throughout the brain according to the level of perfusion. Once in brain cells, the tracer changes, becoming hydrophilic (for HMPAO this process is thought to involve glutathione, for ECD a peroxidase mediated change). It cannot therefore exit neurones, and cannot be washed out or redistributed.

This means that the distribution in brain of the tracer, and the radionuclide attached to it, continues to reflect regional cerebral perfusion as it was at the time of the injection, even if the scan is carried out some time later. The injection can be given during the seizure, and the patient can then be allowed to recover and scanned later when able to co-operate.

In fact, tracer takes approximately 15 seconds to reach the brain after intravenous injection, and uptake into the brain takes approximately 40 seconds. The image acquired is therefore that of the average cerebral perfusion during a period of 40 seconds, which begins 15 seconds after the injection. The kinetics of the tracer therefore limits both the temporal resolution and the sampling of the technique, factors which are crucial to appropriate image interpretation.
Underlying the studies of seizures which have been carried out over the past two decades is the assumption that changes in perfusion are secondary to, and reflect, changes in the local level of neuronal electrical activity. A large body of evidence closely links the two in normal physiological situations, but there is some reason to doubt that the link is inviolate during seizures, where energy demand is so far in excess of that seen in physiological situations. Recent evidence has suggested that ictal hyperperfusion will only continue for a certain period of time in some brain regions, even if the ictal discharge carries on [19], again suggesting that perfusion declines relative to the level of neuronal activity.

Peri-ictal rCP SPECT is part of presurgical evaluation at a number of centres, mainly in Australia, Europe and the USA. It is usually carried out with surface EEG monitoring, so that patients who have peri-ictal SPECT will also have ictal EEG recordings, and usually specialised MRI studies. In most medial temporal lobe epilepsies this combination of non-invasive investigations will give sufficient information either to proceed to surgery or to determine that the patient is not a surgical candidate. Increasing weight is being given to MRI findings in presurgical investigation, but some MRI negative patients who have typical ictal rCP SPECT findings go on to have good surgical outcomes [20]. In extratemporal epilepsies, ictal SPECT data can be useful in deciding on the placement of intracranial, particularly if no lesion is detected on MRI.

\subsection{The ictal phase}

\section{Medial temporal lobe seizures}

Medial temporal lobe seizures are the most common focal seizures in adults, and respond well to surgical treatment. Much of the ictal and postictal SPECT literature, therefore, relates to such seizures.

In short medial temporal seizures, the typical ictal pattern is seen if injection of tracer is carried out during the seizure discharge or up to 15 seconds after its termination. Recent data suggests that if the seizure discharge lasts more then 100 seconds, then the pattern of perfusion becomes 'postictal' despite continuation of the discharge [19].

The typical ictal pattern (Fig. 1) consists of hyperperfusion of the whole temporal lobe, which can often be very marked, with HMPAO uptake up to twice that in the contralateral temporal lobe. This may be accompanied by hypoperfusion of surrounding cortical struc- 


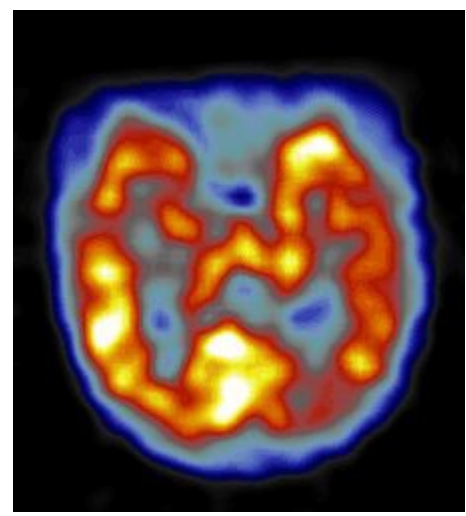

Fig. 1. SPECT scan following HMPAO injection during a complex partial seizure originating in the left mesial temporal lobe. The slice is in the plane of the long axis of the temporal lobe, and shows hyperperfusionof the left temporal lobe, mainly involving the mesial cortex and pole (more typically, hyperperfusion extends posteriorly into the lateral temporal cortex). There is hypoperfusion of the ipsilateral occipital cortex.

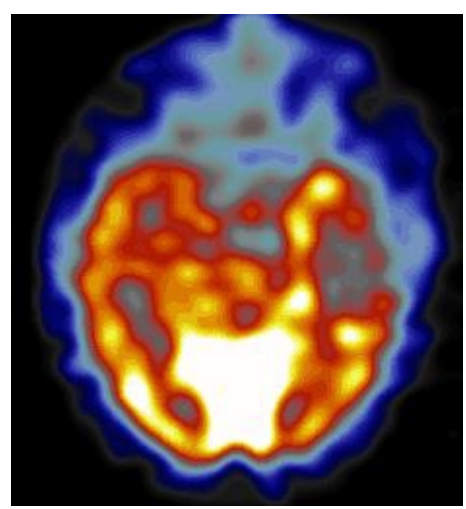

Fig. 2. SPECT scan following injection of HMPAO 1 minute after termination of a complex partial seizure originating in the left mesia temporal lobe. The slice is in the plane of the long axis of the temporal lobe. There is hyperperfusion of the left mesial temporal cortex extending toward the temporal pole, with lateral temporal hypoperfusion. Relatively high perfusion in the mesial occipital cortex is usual, as patients usually have their eyes open during complex partial seizures.

tures, usually the orbital cortex, the ipsilateral frontal lobe or the whole ipsilateral hemisphere [21-25].

The fact that areas outside the epileptogenic zone are hyperperfused is thought to be due either to secondary activation by seizure discharges conducted through normal white matter pathway, or by direct invasion of neighbouring structures by the seizure discharge itself. The lateral temporal cortex is almost always hyperperfused, as is the insular cortex, but other structures such as the ipsilateral orbital cortex, the ipsilateral motor cortex and the basal ganglia are also commonly involved.

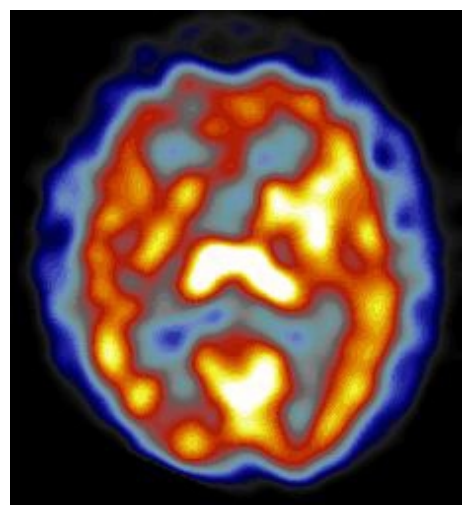

Fig. 3. SPECT following HMPAO injection during a left frontal lobe seizure. This axial slice shows hyperperfusion of the left insular and lateral temporal cortex, with hyperperfusion of the adjacent basal ganglia. The contralateral frontal cortex is hypoperfused.

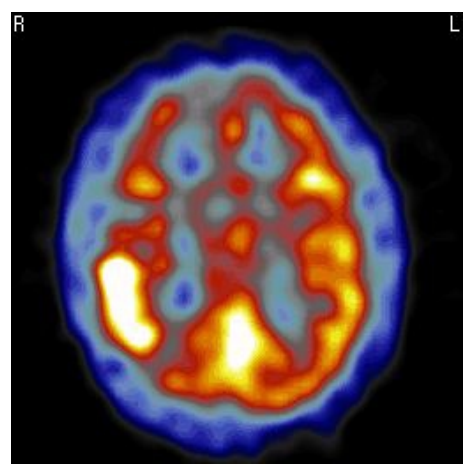

Fig. 4. SPECT scan following injection of HMPAO near onset of a right parietal lobe seizure. The axial slice shows an intense focus of hyperperfusion in the right parietal lobe, accompanied by hypoperfusion of the resto of the ipsilateral hemisphere.

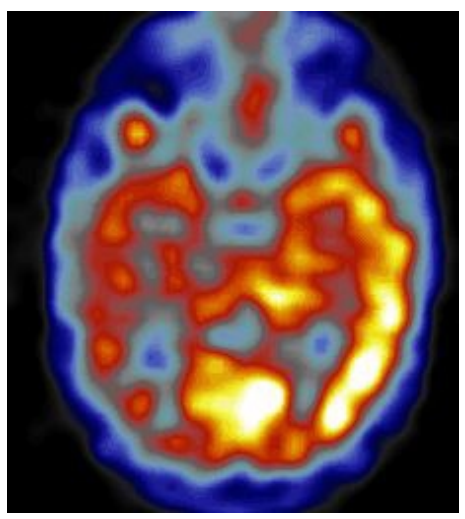

Fig. 5. SPECT scan following injection of HMPAO 40 seconds after the onset of hemparesis during the Wada test. The slice is in the plane of the long axis of the temporal lobe. Left carotid injection. The slice is oriented in the long axis of the temporal lobe and shows hypoperfusion of temporal structures, in this case including medial temporal cortex. 
In some patients, hyperperfusion extends posteriorly into the lateral temporal cortex, and in a minority of patients bilateral changes are seen.

Some correlation between the pattern of hyperperfusion and the clinical appearance of the seizure may be made: for example hyperperfusion of the basal ganglia has been associated with contralateral tonic posturing during the seizure [26]. Patterns of perfusion seen during temporal lobe seizures have also been related to operative outcome [20]. The typical pattern described above, a bilateral pattern, and the typical pattern with posterior extension of hyperperfusion were all associated with good outcome, whereas atypical patterns were associated with lack of pathology and poor outcome (presumably because atypical rCP patterns were associated with epileptogenesis outside the field of the excision).

Injection during secondary generalised seizures may show localising rCP changes, particularly if the seizure has had a clear focal onset. Injection must be early, however: if it is carried out in the clonic phase, it is the author's experience that a bilateral postictal pattern (see below) is usually seen, giving localising, but not lateralising, information. Ictal SPECT seems to show similar findings in medial temporal lobe seizures in children $[27,28]$ as in adults.

\subsection{The postictal phase}

In the immediate postictal phase ( $0-4$ minutes after the end of the ictal discharge), the lateral temporal cortex rapidly becomes markedly hypoperfused. This often extends into the ipsilateral frontal lobe, or hemisphere [23-25,29,30]. Occasionally, areas of hypoperfusion may be seen in the contralateral hemisphere, and this may cause difficulty in interpretation. During this early postictal period, the medial temporal cortex remains hyperperfused, later (4-15 minutes) becoming isoperfused relative to the contralateral medial temporal cortex. As time goes on, it too becomes hypoperfused, but even relatively late into the postictal period, there is usually some preservation of medial perfusion relative to the lateral perfusion.

Approximately $20 \%$ of patients injected in the postictal period will show bilateral and approximately symmetric changes [29,30] (Fig. 2). This provides some supporting evidence of medial temporal lobe origin for the seizure, but no lateralising evidence. Where the changes are asymmetric, the side of predominance is not always the side of seizure origin. Bilateral or non-typical postictal perfusion patterns should be interpreted cautiously.

The sensitivity of peri-ictal SPECT in medial temporal epilepsies depends on the timing of the injection. Ictal injections show changes in almost all cases, although bilateral changes may prevent lateralisation. Postictal injections give a sensitivity of $70-90 \%$, depending on the time between the end of the seizure and the injection $[29,30]$. With ictal injection, the specificity appears to be close to $100 \%$, provided the perfusion pattern is typical.

\section{Non medial temporal lobe seizures}

A number of reports describing rCP during seizures originating outside medial temporal structures have been published [31-37], showing patterns of perfusion clearly different from those seen during medial temporal seizures.

\subsection{The temporo-parieto-occipital junction (TPOJ)}

TPOJ seizures have clinical and electrographic characteristics which suggest origin in the posterolateral cortex plus variable propagation into the (usually) ipsilateral temporal lobe. Such seizures do appear to be distinct in terms of ictal SPECT findingsc[35]. TPOJ seizures are associated with marked hyperperfusion in the area of seizure onset. This may be an isolated change, but there may also be a variable degree of hyperperfusion of anterolateral temporal lobe structures, with medial temporal structures being hyperperfused to a lesser degree, or even hypoperfused. In some cases, there is a small area of hyperperfusion in the contralateral parietal lobe, which may correlate with bilateralisation of the seizure discharge.

\subsection{The frontal lobe}

Frontal lobe seizures are often short, presenting technical limitations to the use of SPECT. The use of a pre-mixed tracer will be necessary to capture seizures lasting less than 30 seconds, even when the person injecting is standing over the patient waiting for a seizure. Seizures lasting much less than this will be difficult to capture. Postictal injection is usually of no value.

Frontal lobe seizures are associated with a variety of ictal changes [31,33,34,36] (Fig. 3), distinct from those seen in medial temporal seizures. In cases published so far, ictal perfusion changes appear to be consistent 
with the site of origin of the seizure within the frontal lobe, where this is known, although in one case changes were seen distant from the site of origin indicated by EEG, albeit within the 'correct' frontal lobe [36]. However, hyperperfusion may be widespread or bilateral, limiting the localising information gained $[31,36]$. Hyperperfusion of subcortical structures such as the basal ganglia, thalamus and cerebellum is common $[31,36]$.

Ictal hypoperfusion is also common, and is variable in pattern and location. Hemispheric hypoperfusion may be either ipsilateral or contralateral to ictal hyperperfusion [31,36], and is often prominent posteriorly.

\subsection{The parietal lobe}

Little has been published, but pure parietal lobe seizures seem to be associated simply with localised hyperperfusion in the parietal lobe (Fig. 4), if any changes are seen at all [32].

\subsection{Occipital lobe seizures}

One small series is published [37]. Occipital lobe seizures seem to be in some senses similar to TPOJ seizures (presumably due to similarities in the pattern of propagation of the seizure discharge), in that some have hyperperfusion of one occipital lobe, while others also show changes in the ipsilateral temporal lobe. Temporal lobe changes have consisted of hyperperfusion of medial structures, with lateral hypoperfusion.

\section{Interpretation and analysis of peri-ictal $\mathrm{rCBF}$ images}

Interpretation of ictal or postictal rCP changes requires formal comparison of datasets, and can usefully be separated into stages:

1. Determination of interictal and ictal perfusion patterns.

2. Determination of the change that has taken place.

3. Explaining the change by developing a hypothesis of seizure origin and spread.

Numeric analysis of ictal SPECT images has been used mainly in research contexts. Measurements of count densities in regions of interest have to be related to reference areas to allow comparisons between two separate scans. Measurements of mean count density in a region of interest can be related to the same measurement in a homotopic structure, in a distant reference structure, or to whole brain count density. However, all such techniques assume that the reference area is not involved in the physiological changes to be measured, an assumption which is not at all warranted in this situation. Bilateral changes are common on ictal datasets, as are changes involving a variety of subcortical and subtentorial structures, and changes involving the whole of a hemisphere (which will significantly affect mean count density for the whole brain). Statistical parametric mapping [38] can statistically compare patterns of perfusion with the average pattern of a database of normal datasets, and can overcome many of the disadvantages older techniques. Formal co-registration and subtraction of images [39] can be useful in defining ictal hyperperfusion which takes place at the margin of an area which is hypoperfused interictally, but its application and interpretation requires care and experience. Co-registration of SPECT datasets with MRI is mandatory for the use of most numeric techniques and for subtractions (and improves the precision of visual interpretation).

\section{Neurotransmitter receptor function}

PET has been more widely used than SPECT for imaging receptors in epilepsy, although two SPECT neuroreceptor ligands have been applied to epilepsy. There is a SPECT ligand for the GABA receptor associated benzodiazepine (BZ) site, ${ }^{123}$ I-iomazenil. Several small studies show focal reductions in binding, but there is some evidence that false localisations occur [40]. Reduced BZ binding probably correlates with cell loss in the medial temporal lobe $[41,42]$, and may simply be a sensitive marker for it. Muscarinic receptors have been imaged in medial temporal lobe epilepsies using ${ }^{123}$ I dexetamide, showing focal reduction in binding $[43,44]$.

\section{SPECT imaging during the intracarotid amytal test}

The Wada (intracarotid amytal) test is widely used to assess speech and memory function as part of the presurgical work-up for temporal lobe surgery. A catheter is placed in the internal carotid artery, and sodium amytal is injected, perfusing and transiently rendering non-functional structures in the distribution of the middle cerebral artery. This produces a contralateral hemiparesis, and aphasia when the dominant 
side is injected, as well as impairment of material specific memory function. The results are used to lateralise speech function and to predict the occurrence of an amnesic syndrome following temporal lobe surgery.

While the test appears to work, the mechanism has not been clear. The hippocampus is usually perfused through the posterior cerebral artery, and so intracarotid injection of amytal would not therefore be expected to affect memory (Fig. 5). The distribution of amytal during the Wada test can be imaged using SPECT, by injecting HMPAO intra-arterially through the catheter in the internal carotid artery. To define the distribution of brain structures rendered hypofunctional by the amytal, a normal dose of HMPAO is injected intravenously 30-60 seconds after the onset of the hemiparesis [45, 46], and acquisition of the image is carried out in the normal way after the amytal test is finished.

Initial studies suggested that, as one might expect from the anatomy, amytal injected into the carotid artery does not reach the hippocampus, and has no effect on its perfusion and, by extension, its function. It had been suggested that the effect of the amytal extended into structures not perfused by it, by a diaschisis type mechanism. However, the use of a double intravenous/intracarotid injection and acquisition technique has shown that the distribution of amytal matches that of hypoperfusion in the medial temporal lobe, though mismatches are seen in other areas [47]. The use of high resolution SPECT combined with slice orientation in the plane of the temporal lobe shows that a partial reduction in perfusion is seen in the great majority of patients [47]. Given that memory is a relatively sensitive function, such a partial effect is probably sufficient to explain the action of the test.

\section{References}

[1] F.A. Gibbs and W.G. Lennnox, EL Gibbs Cerebral blood flow preceding and accompanying seizures in man, Archives of Neurology \& Psychiatryry 32 (1934), 257-272.

[2] W. Penfield, The circulation of the epileptic brain Research, Publication of the Association for Nervous and Mental Disease 18 (1937), 605-737.

[3] W. Penfield and K. von Santha, A Cipriani Cerebral blood flow during induced epileptiform seizures in animals and man, Journal of Neurophysiology 2 (1939), 257-267.

[4] F.J. Bonte, M.D. Devous, E.M. Stokely and R.W. Homan, Single photon tomographic determination of regional cerebral blood flow in epilepsy, American Journal of Neuroradiology 4 (1983), 544-546.

[5] I. Podreka, W. Lang, E. Suess, D. Wimberger, M. Steiner, W. Gradner, M. Zeitlhofer, G. Pelzl, B. Mamoli and L. Deecke, Hexamethyl-propylene-amine-oxime (HMPAO) single photon emission computed tomography (SPECT) in epilepsy, Journal of Brain Topography 1 (1988), 55-60.
[6] R. Duncan, J. Patterson, D.M. Hadley, P. Macpherson, M.J. Brodie, I. Bone, A.P. McGeorge and D.J. Wyper, CT, MR and SPECT imaging in temporal lobe epilepsy, Journal of Neurology Neurosurgery and Psychiatry 53 (1990), 11-15.

[7] R. Duncan, J. Patterson, D.M. Hadley, I. Bone, A.P. McGeorge and D.J. Wyper, Tc99m HMPAO single photon emission computed tomography in temporal lobe epilepsy, Acta Neurologica Scandinavica 81 (1990), 287-293.

[8] C.C. Rowe, S.F. Berkovic, M.C. Austin, M. Saling, R.M. Kalnins, W.J. McKay and P.F. Bladin, Visual and quantitative analysis of interictal SPECT with Tc99m HMPAO in temporal lobe epilepsy, Journal of Nuclear Medicine 32 (1991), 16881694.

[9] S. Duncan, J. Gillan, R. Duncan and M. Brodie, Interictal HMPAO SPECT: a routine investigation in medically intractable complex partial epilepsy? Epilepsy Res 13 (1992), 83-87.

[10] R. Duncan, J. Patterson, D.M. Hadley, R. Roberts and I. Bone, Interictal temporal hypoperfusion is related to early onset temporal lobe epilepsy, Epilepsia 37 (1996), 134-140.

[11] P. Ryvlin, L. Garcia-Larrea, B. Phillipon, J.C. Froment, J.C. Fischer, Revol, M. and Mauguiere, F., High signal intensity on T2 weighted MRI correlates with hypoperfusion in temporal lobe epilepsy, Epilepsia 33 (1992), 28-35.

[12] B. Guillon, R. Duncan, A. Biraben, A.M. Bernard, Vignal, J.P. and P. Chauvel, Correlation between interictal regional cerebral blood flow and depth recorded interictal spiking in temporal lobe epilepsy, Epilepsia 39 (1998), 76-76.

[13] G.S. Cruickshank, in: SPECT imaging of the brain, R. Duncan, ed., Kluwer, Dordrecht, 1997, pp. 161-178.

[14] W. Lang, I. Podreka, E. Suess, C. Muller and L. Deecke, Single photon emission computed tomography during and between seizures, Journal of Neurology 235 (1988), 277-284.

[15] A.S. Harvey, I.J. Hopkins, J.M. Bowe, D.J. Cook, L.K. Shield and S.F. Berkovic, Frontal lobe epilepsy: clinical seizure characteristics and localisation with ictal 99mTc HMPAO SPECT, Neurology 43 (1993), 1966-1980.

[16] R. Duncan, J. Patterson, D.M. Hadley and R. Roberts, Ictal regional cerebral blood flow in frontal lobe seizures, Seizure 6 (1997), 393-401.

[17] A.R. Andersen, H. Friberg, J.F. Schmidt and S.G. Hasselbalch, Quantitative measurements of cerebral blood flow using SPECT and Tc99m D,L- HMPAO compared to xenon-133, Journal of Cerebral Blood Flow \& Metabolism 8(1) (1988), S69-81.

[18] L. Friberg, A.R. Andersen, N.A. Lassen, S. Holm and M. Dam, Retention of $99 \mathrm{mTc}$ bicisate in the human brain after intracarotid injection, Journal of Cerebral Blood Flow \& Metabolism 14(1) (1994), S19-27.

[19] R.A. Avery, S.S. Spencer, M.V. Spakani, M. Corsi, J.P. Seibyl and I.G. Zubal, Effect of injection time on postictal SPET persusion changes in medically refractory epilepsy European, Journal of Nuclear Medicine 26 (1999), 830-836.

[20] S.S. Ho, M.R. Newton, A.M. McIntosh, R.M. Kalnins, G.C. Fabinyi, G.A. Brazenor, W.J. McKay, P.F. Bladin and S.F. Berkovic, Perfusion patterns during temporal lobe seizures: relationship to surgical outcome, Brain 120 (1997), 19211928.

[21] B.I. Lee, O.N. Markand, H.N. Wellman, A.R. Siddiqui, H.M., Park, B. Mock, R.M. Worth, M.K. Edwards and J. Krepshaw, HIPDM SPECT in patients with medically intractable complex partial seizures, Archives of Neurology 45 (1988), 397-402.

[22] W. SheN, B.I. Lee, H.M. Park, A.R. Siddiqui, H.H. Wellman, R.M. Worth and O.M. Markand, HIPDM-SPECT brain imag- 
ing in the presurgical evaluation of patients with intractable seizures, Journal of Nuclear Medicine 31 (1990), 1280-1284.

[23] M.R. Newton, S.F. Bercovik, M.C. Austin, C.C. Rowe, W.J McKay and P.F. Bladin, Postictal switch in blood flow distribution and temporal lobe seizures, Journal of Neurology Neurosurgery and Psychiatry 55 (1992), 891-894.

[24] R. Duncan, J. Patterson, R. Roberts, D.M. Hadley and I. Bone, Ictal/postictal SPECT in the pre-surgical localisation of complex partial seizures, Journal of Neurology Neurosurgery and Psychiatry 56 (1993), 141-148.

[25] S.S. Ho, S.F. Berkovic, W.J. Kay, R.M. Kalnins and P.F. Bladin, P.F., Temporal lobe epilepsy subtypes: differential patterns of cerebral perfusion seen on ictal SPECT, Epilepsia 37 (1996), 788-795.

[26] M.R. Newton, S.F. Berkovic and Austen, M.C., Dystonia, clinical lateralisation and regional blood flow changes in temporal lobe seizures, Neurology 42 (1992), 371-377.

[27] Harvey, A.S., Bowe, J.M., Hopkins, I.J., Shield, L.K., Cook, D.J. and S.F. Berkovic, Ictal 99mTc HMPAO single photon emission computed tomography in children with temporal lobe epilepsy, Epilepsia 34 (1993), 869-877.

[28] Cross, J.H., Boyd, S.G., Gordon, I., Harper, A. and Neville, B.G., Ictal cerebral perfusion related to EEG in drug resistant focal epilepsy of childhood, Journal of Neurology Neurosurgery and Psychiatry 62 (1997), 377-384.

[29] C.C. Rowe, Bercovic, S.F., Sia, S.T.B., Austin, M., McWJ Kay, R.M. Kalnins and P.F. Bladin, P.F., Localisation of epileptic foci with postictal single photon emission computed tomography, Annals of Neurology 26 (1989), 660-668.

[30] C.C. Rowe, S.F. Berkovic, S.T.B. Sia and P.F. Bladin, Patterns of postictal blood flow in temporal lobe epilepsy: qualitative and quantitative findings, Neurology 41 (1991), 1096-1103.

[31] A.S. Harvey, I.J. Hopkins, J.M. Bowe, D.J. Cook, L.K. Shield and S.F. Berkovic, Frontal lobe epilepsy: clinical seizure characteristics and localisation with ictal 99mTc HMPAO SPECT, Neurology 43 (1993), 1966-1980.

[32] S.S. Ho, S.F. Berkovic, M.R. Newton, M.C. Austin, W.J. Mc Kay and P.F. Bladin, Ictal 99mTc HMPAO SPECT findings in parietal lobe epilepsy, Epilepsia 34(2) (1993), 112.

[33] D.A. Marks, A. Katz, P. Hoffer and S.S. Spencer, Localisation of extratemporal epileptic foci during ictal single photon emission computed tomography, Annals of Neurology 31 (1992), 250-253.

[34] M.R. Newton, S.F. Berkovic, M.C. Austin, C.C. Rowe, McWJ Kay and P.F. Bladin, SPECT in the localisation of extratemporal and temporal seizure foci, Journal of Neurology Neurosurgery \& Psychiatry 59 (1995), 26-30.

[35] R. Duncan, S. Rahi, A.M. Bernard, A. Biraben, A. Devillers, J. Lecloirec, J.P. Vignal and P. Chauvel, Ictal cerebral blood flow in seizures originating in the posterolateral cortex, Journal of Nuclear Medicine 37 (1996), 1946-1951.

[36] R. Duncan, J. Patterson, D.M. Hadley and R. Roberts, Ictal regional cerebral blood flow in frontal lobe seizures, Seizure 6 (1997), 393-401.

[37] R. Duncan, J. Patterson, D. Hadley, A. Biraben, A.M. Bernard, A. Devillers, J.P. Vignal and P. Chauvel, Ictal SPECT in occipital lobe seizures, Epilepsia 38 (1997), 839-843.

[38] J.J. Friston, C.D. Frith, P.F. Liddee, R.J. Dola, A.A. Lammrtsma and R.S.J. Frackoviack, The relationship between global and local changes in PET scans, Journal of Cerebral Blood Flow \& Metabolism 10 (1990), 458-466.

[39] I.G. Zubla, S.S. Spencer, K. Imam, J. Seibyl, E.O. Smith, G. Wisniewski and P.B. Hoffer, Difference image calculated from ictal and interictal technetium-99m-HMPAO SPECT scans of epilepsy, Journal of Nuclear Medicine 37 (1996), 1080-1083.

[40] A.C. van Huffelen, J.W. van Isselt and C.W. van Veelen, Identification of the side of the epileptic focus with ${ }^{123}$ I Iomazenil SPECT: a comparison with 18 FDG and ictal EEG findings in patients with medically intractable complex partial seizures, Acta Neurochirurgica Wien 50(1) (1990), 95-99.

[41] J.W. McDonald, E.A. Garofalo, T. Hood, J.C. Sackellares, S. Gilman, P.E. McKeever, J.C. Troncoso and M.V. Johnson, Altered excitatory and inhibitory amino acid receptor binding in hippocampus of patients with temporal lobe epilepsy, Annals of Neurology 29 (1991), 529-541.

[42] M.J. Koepp, M.P. Richardson, D.J. Brooks, J.B. Poline, K.J. Friston and V.J. Cunningham, Central benzodiazepine receptors in hippocampal sclerosis: an objective in vivo analysis, Brain 119 (1996), 1677-1687.

[43] C.C. Rowe, K.L. Boundy, L. Kitchener, M. Barnden, M. Kassiou, M. Katsifis and R. Lambrecht, Ictal 99mTc HMPAO SPECT and ${ }^{123}$ I-iododexetamide SPECT in temporal lobe epilepsy, in: SPECT in neurology and psychiatry, P. DeDeyn, R.A. Dierckx, A. Alavi and B.A. Pickut, eds., Libbey, London, 1997, pp. 219-224.

[44] H.W. Muller Gartner, J.M. Links, J.L. Prince, R.N. Bryan, E. McVeigh and J.P. Leal, Decreased hippocampal muscarinic choline receptor binding measured by 132I iododexetimide and single photon emission computed tomography in epilepsy, Annals of Neurology 34 (1993), 235-238.

[45] E. Ryding, H. Sjoholm, H. Skeidsvoll and D. Elmqvist, Delayed decrease in hemispheric cerebral blood flow during Wada test demonstrated by $99 \mathrm{mTc}-\mathrm{HMPAO}$ single photon emission computed tomography, Acta Neurologica Scandinavica 80 (1989), 248-254.

[46] P.J. Jeffery, L.H. Monsein, S. Szabo, J. Hart, R.S. Fisher, R.P. Lesser, G.M. Debrun, B. Gordon, H.M. Wagner and E.E. Camargo, Mapping the distribution of amobarbital sodium in the intracarotid Wada test by use of Tc99m HMPAO with SPECT, Radiology 178 (1991), 847-850.

[47] R. De Silva, R. Duncan, J. Patterson, R. Gillham and D. Hadley, Regional cerebral perfusion during the Wada test, Journal of Nuclear Medicine 40 (1999), 747-752. 


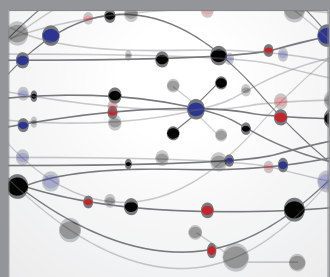

The Scientific World Journal
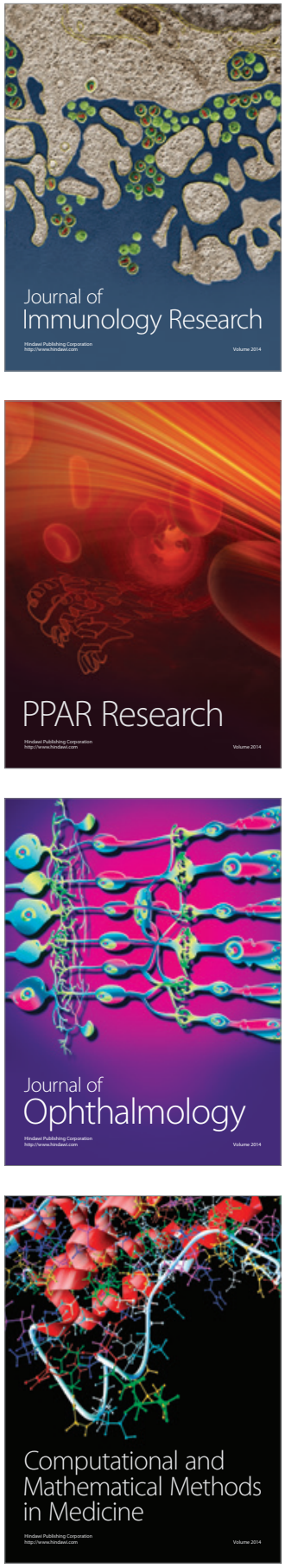

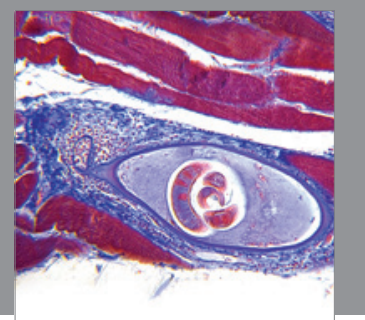

Gastroenterology

Research and Practice
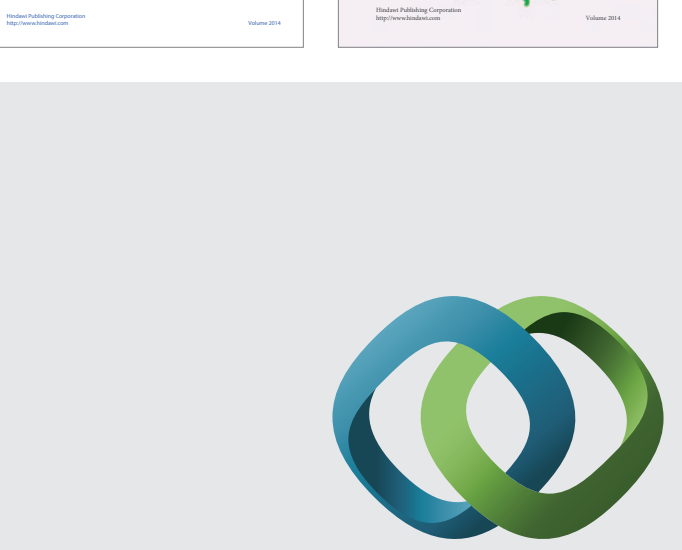

\section{Hindawi}

Submit your manuscripts at

http://www.hindawi.com
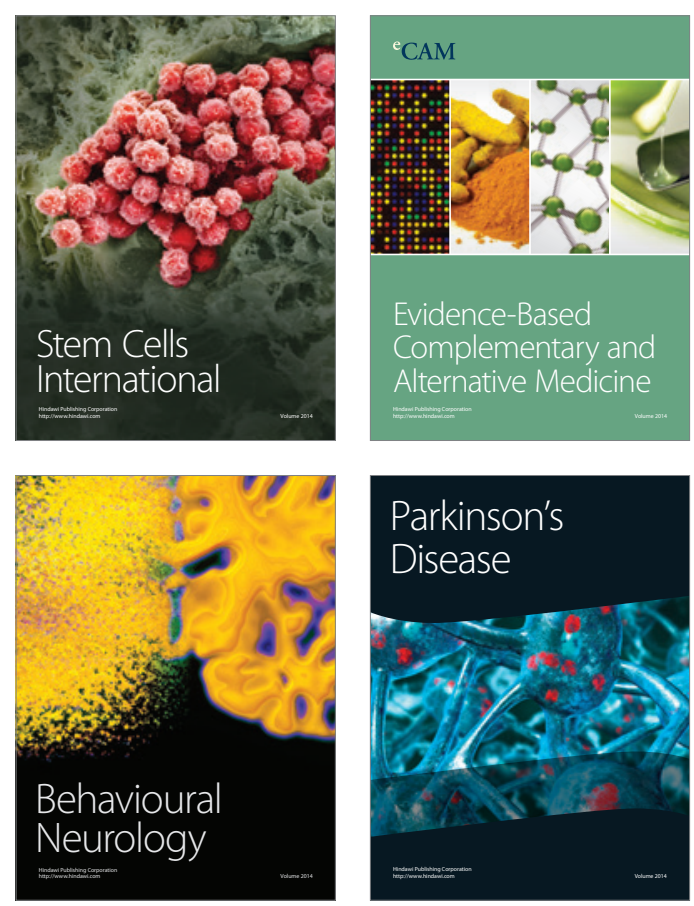

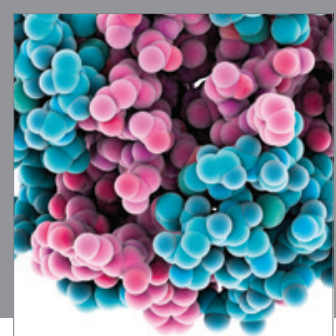

Journal of
Diabetes Research

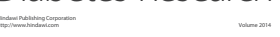

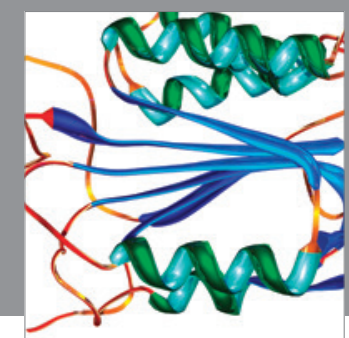

Disease Markers
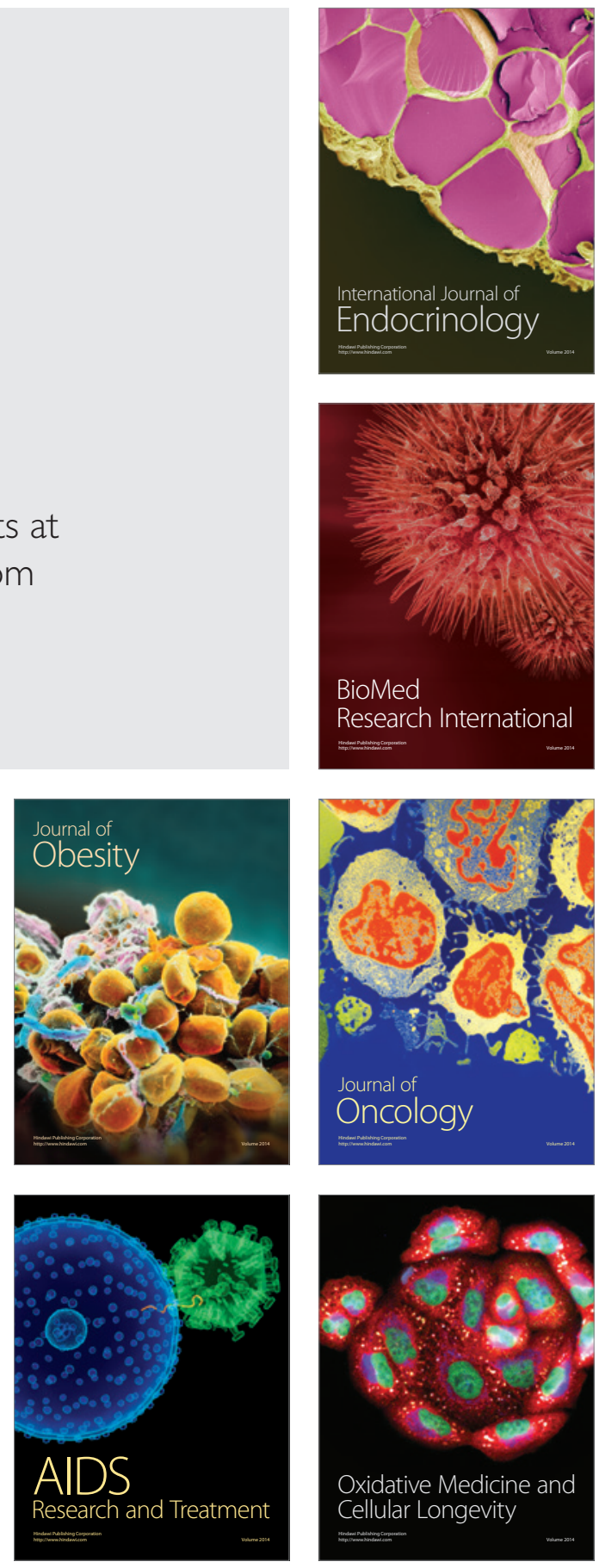